\title{
Inclusão de farelo de gérmen de milho desengordurado na alimentação de frangos de corte ${ }^{1}$
}

\author{
Sandra Regina Brunelli ${ }^{2}$, João Waine Pinheiro ${ }^{3}$, Caio Abércio da Silva ${ }^{3}$, Nilva Aparecida \\ Nicolao Fonseca ${ }^{3}$, Dássia Daiane de Oliveira ${ }^{4}$, Gianne Evans Cunha ${ }^{4}$, Lílian Francisco \\ Arantes de Souza ${ }^{4}$
}

\author{
1 Parte da dissertação de Mestrado da primeira autora. \\ 2 Mestre em Ciência Animal pela UEL - Londrina - PR. \\ ${ }^{3}$ Departamento de Zootecnia - CCA - UEL - Londrina - PR. \\ ${ }^{4}$ Iniciação Científica do Departamento de Zootecnia - CCA - UEL - Londrina - PR.
}

RESUMO - Dois ensaios foram realizados para avaliação do uso de farelo de gérmen de milho desengordurado (FGMD) na alimentação de frangos de corte. No primeiro ensaio, para determinação do valor nutricional pelo método de coleta total de excretas, foram utilizados 120 pintos de corte com 19 dias de idade, distribuídos em delineamento inteiramente casualizado, com três tratamentos e quatro repetições de dez aves por unidade experimental. As dietas experimentais, à base de milho e farelo de soja, variaram de acordo com os níveis de FGMD (0, 20 e 40\%). Os valores médios dos coeficientes

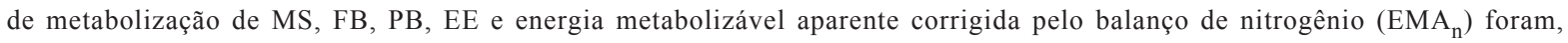
com base na matéria natural (MN), 60,99; 16,73; 53,80 e $82,71 \%$ e $2.413 \mathrm{kcal} / \mathrm{kg}$, respectivamente. No segundo ensaio, foram utilizados 480 pintinhos de quatro dias de idade, distribuídos em um delineamento experimental inteiramente casualizado, com cinco tratamentos e oito repetições (quatro de cada sexo) de 12 aves por unidade experimental. Os tratamentos consistiram de dietas experimentais à base de milho e farelo de soja, com 0, 5, 10, 15 e 20\% de FGMD. Foram observados efeitos linear crescente do FGMD para ganho de peso (GP) e consumo de ração (CR) e linear decrescente para conversão alimentar (CA). A inclusão de $20 \%$ de FGMD proporcionou efeitos positivos nas características de desempenho de frangos de corte e não alterou as características de carcaça.

Palavras-chave: alimentos, aves, desempenho, energia metabolizável, nutrição

\section{Feeding increasing defatted corn germ meal levels to broiler chickens}

\begin{abstract}
Two trials were carried out to evaluate the effects of feeding of increasing defatted corn germ meal (DCGM) levels to broilers. In the first trial, for determination of nutritional value by method of total excreta collection, 120 19-day old broilers were allotted to a completely randomized design with three treatments and four replications of 10 birds each. The treatments consisted of corn and soybean meal-based diets with increasing DCGM levels: 0, 20, and 40\%. Mean values of coefficients of metabolization of DM, CF, CP, EE, and metabolizable energy adjusted for nitrogen balance were (as-fed basis): $60.99 \%, 16.73 \%, 53.80 \%, 82.71 \%$, and $2413 \mathrm{kcal} / \mathrm{kg}$, respectively. In the performance trial, 480 4-day old chicks were assigned to a completely randomized design with five treatments and eight replications (four of each sex) of 12 birds. The treatments consisted of corn and soybean meal-based diets with increasing DCGM levels: 0, 5, 10, 15, and 20\%. Linear increasing effect of DCGM on weight gain and feed intake and decreasing linear effect on feed:gain ratio were observed. Feeding DCGM is viable up to $20 \%$ level to broiler performance with no changes on carcass traits.
\end{abstract}

Key Words: food, metabolizable energy, nutrition, performance, broiler

\section{Introdução}

Na avicultura, são freqüentes os períodos de instabilidade financeira advindos dos altos preços dos ingredientes que compõem as rações. A alimentação dos frangos de corte sustenta-se basicamente da utilização do milho e do farelo de soja e qualquer variação nos seus preços de mercado reflete diretamente nos resultados econômicos da atividade.
Segundo Butolo et al. (1998), há um interesse crescente por alimentos alternativos para aves.

Entre as alternativas alimentares pesquisadas para frangos, estão os resíduos e os co-produtos das agroindústrias, como o gérmen de milho desengordurado, por exemplo. Segundo Rostagno (2003), o farelo de gérmen de milho (com ou sem óleo) tem sido pesquisado como sucedâneo do milho nas rações de monogástricos. 
O farelo de gérmen de milho desengordurado (FGMD) é resultante do processamento industrial do milho para extração do óleo do gérmen, que, depois de retirado da semente na moagem úmida, é seco e prensado (Andriguetto et al., 1983; Dermachi, 1998).

A composição química do FGMD observada na literatura é divergente. Estudos realizados no Brasil relatam valores médios de $10,7 \%$ de $\mathrm{PB}, 3,9 \%$ de FB, $1,2 \%$ de EE e $3,4 \%$ de CIN e uma quantidade interessante de $\mathrm{K}(0,77 \%)$, $\mathrm{Mg}(0,51 \%), \mathrm{Fe}$ (108ppm) e Zn (72 ppm) (Butolo et al., 1998; Brum et al., 1999; Rodrigues et al., 2001ab).

Brum et al. (1999) determinaram, em frangos de corte, valor energético do FGMD de 2.468 ( \pm 39$) \mathrm{kcal}$ de EMAn $/ \mathrm{kg}$ de $M N$, enquanto Butolo et al. (1998) estimaram valor de $2.392 \mathrm{kcal}$ de EMA/ $\mathrm{kg}$ de MN e $2.309 \mathrm{kcal}$ de EMAn/ $\mathrm{kg}$ de MN. Rodrigues et al. (2001a), por sua vez, encontraram $2.207 \mathrm{kcal}$ de EMAn/ $\mathrm{kg}$ de MN e energia metabolizável verdadeira corrigida de $2.267 \mathrm{kcal} / \mathrm{kg}$ de $\mathrm{MN}$ com frangos de corte.

Os parâmetros zootécnicos que indicam a quantidade de alimento a ser utilizada em uma dieta não são bem definidos. Conseqüentemente, são desconhecidos também os valores ideais para obtenção de melhores resultados de desempenho e produtividade dos frangos.

Este trabalho foi realizado com o objetivo de avaliar o uso do farelo de gérmen de milho desengordurado em rações para frangos de corte.

\section{Material e Métodos}

Dois ensaios foram conduzidos no Setor de Avicultura da Fazenda Escola da Universidade Estadual de Londrina, em Londrina, PR.

No primeiro ensaio, para o estudo da digestibilidade, pelo método tradicional de coleta total de excretas (Albino et al., 1982), foram utilizados 120 pintos de corte de 19 dias de idade, da linhagem Cobb, sexados, alojados em uma bateria metálica suspensa, distribuídos em um delineamento experimental inteiramente casualizado, com três tratamentos e quatro repetições de dez aves (metade de cada sexo).

Os tratamentos experimentais consistiram de uma ração-referência, à base de milho e farelo de soja (Tabela 1), formulada para atender às exigências nutricionais das aves, conforme descrito por Rostagno et al. (2000), e de outras duas rações, em que o farelo de gérmen de milho desengordurado substituiu, com base na matéria natural, 20 e $40 \%$ da ração-referência.

O período de ensaio constituiu-se de nove dias, quatro para adaptação das aves às gaiolas e às rações experimentais e cinco para coleta das excretas.
As excretas foram coletadas duas vezes ao dia e armazenadas em congelador até o final do ensaio, quando foram descongeladas, devidamente reunidas por tratamentos, homogeneizadas e pesadas. Posteriormente, duas amostras de cada tratamento foram retiradas e secas em estufa ventilada por 72 horas a $55^{\circ} \mathrm{C}$

As análises das rações e das excretas foram realizadas no Laboratório de Tecnologia de Alimentos e Medicamentos (TAM) da Universidade Estadual de Londrina (UEL), de acordo com as normas do Instituto Adolfo Lutz (1985). As concentrações de EB das amostras das rações, dos alimentos e das excretas foram determinadas por meio de bomba calorimétrica, no Laboratório de Nutrição Animal da EMBRAPA - Suínos e Aves de Concórdia, SC.

Para determinação dos valores de EM e dos coeficientes de digestibilidade aparente do FGMD, adotaram-se as equações citadas por Matterson et al. (1965). Os parâmetros analisados neste ensaio foram os coeficientes de metabolização da energia, do EE, da FB, da PB e da MS do gérmen de milho desengordurado.

Tabela 1 - Composição percentual da ração-referência Table 1 - Ingredient composition of the basal diet

\begin{tabular}{lr}
\hline $\begin{array}{l}\text { Ingrediente (\%) } \\
\text { Ingredient }\end{array}$ & $\begin{array}{c}\text { Valores } \\
\text { Values }\end{array}$ \\
\hline Milho (Corn) & 52,502 \\
Farelo de soja (Soybean meal) & 38,081 \\
Óleo de soja (Soybean oil) & 5,713 \\
Fosfato bicálcio (Dicalcium phosphate) & 2,105 \\
Calcário (Limestone) & 0,586 \\
Sal comum (Salt) & 0,303 \\
DL-metionina (99\%) (DL-methionine) & 0,177 \\
L-lisina HCl (79\%) (L-lysine HCl) & 0,033 \\
Suplemento vitamínico + mineral & 0,500 \\
Vitamin + Mineral supplement & 100,00 \\
Total &
\end{tabular}

Valor calculado

Calculated value

Energia metabolizável (kcal/kg) (Metabolizable energy) 3.135

Proteína bruta (\%) (Crude protein) 22,000

Cálcio (\%) (Calcium)

0,900

Fósforo disponível (\%) (Available phosphorus) $\quad 0,500$

Metionina total (\%) (Total methionine) $\quad 0,657$

Metionina+Cistina total (Total methionine + Cystine) $\quad 0,880$

Suplemento vitamínico e mineral por kg de produto: Vit. A $1.400 .000 \mathrm{UI}$ Vit. D3 300.000UI; Vit. E 2.400 mg; Vit. K3 300 mg; Vit. B1 320 mg; Vit. B2 $1.000 \mathrm{mg}$; Vit. B6 $520 \mathrm{mg}$; Vit. B12 $2.000 \mathrm{mg}$; Niacina $7.000 \mathrm{mg}$; Ac. Pantotênico $2.600 \mathrm{mg}$, Ac. Fólico 140 mg; Manganês 15.000 mg; Zinco $10.000 \mathrm{mg}$; Ferro $10.000 \mathrm{mg}$; Cobre $1.600 \mathrm{mg}$; lodo $150 \mathrm{mg}$; Selênio $60 \mathrm{mg}$ Cobalto 40 mg; Metionina 290.000 mg; Colina 98.000 mg; Cocciodiostático $120 \mathrm{~g} ;$ Veículo q.s.q -1000 g

Mineral and vitamin supplement. Nutritional levels per kg of product: Vit. A 1,400,000 IU Vit. $D_{3} 300,000 I U ;$ Vit. E 2, 400 mg; Vit. $K_{3} 300 \mathrm{mg}$; Vit. $B_{1} 320 \mathrm{mg}$; Vit. $B_{2} 1,000 \mathrm{mg}$; Vit. $B$ $520 \mathrm{mg}$ : Vit. $B_{12} 2,000 \mathrm{mg}$ : Niacin 7,000 mg: Pantothenic acid 2,600 mg: Folic acid $140 \mathrm{mg}$ Manganese 15,000 mg; Zinc 10,000 mg; Iron 10,000 mg; Copper 1,600 mg; lodine $150 \mathrm{mg}$ Selenium $60 \mathrm{mg}$; Cobalt $40 \mathrm{mg}$; Methionine 290,000 mg; Choline 98,000 mg; Cocciodiostatic 120 g; vehicle q. s. p. $-1,000 \mathrm{~g}$. 
No segundo ensaio (desempenho), foram utilizados 480 pintos Cobb (50\% de cada sexo), divididos em duas fases ( 4 a 21 e 4 a 42 dias de idade), alojados em um galpão convencional.

Foram avaliados cinco níveis crescentes de $\operatorname{FGMD}(0$, $5,10,15$ e $20 \%$ ) nas rações, formuladas com base nos valores de EMAn e nos resultados da análise bromatológica obtidos no ensaio de digestibilidade do FGMD. A composição de aminoácidos foi calculada utilizando-se os valores obtidos por Soares et al. (2004). Os pintos foram pesados no início do experimento e distribuídos aleatoriamente nos boxes, separados por sexo e tratamento experimental, em um delineamento inteiramente casualizado, em esquema fatorial 5 x 2 (cinco níveis de FGMD e dois sexos), mantendo-se o peso médio do lote por unidade experimental. Cada tratamento teve oito repetições de 12 aves, totalizando 96 aves por tratamento.

O programa de alimentação foi montado considerando as três fases de desenvolvimento dos frangos: ração inicial (4 a 21 dias de idade), ração de crescimento ( 22 a 35 dias de idade) e ração final (36 a 42 dias de idade). As rações experimentais (Tabelas 2, 3 e 4), para cada fase, foram formuladas para atender às exigências mínimas descritas por Rostagno et al. (2000) e foram fornecidas à vontade.

A cada fase experimental e ao término de todo o período experimental, foram avaliados o ganho de peso (GP), o consumo de ração (CR) e a conversão alimentar (CA).

Aos 43 dias de idade, após jejum de 12 horas, foram abatidas 40 aves, uma de cada unidade experimental, com peso médio da unidade experimental, totalizando oito aves por tratamento (metade de cada sexo), para avaliação do peso ao abate, dos pesos e rendimentos de carcaça (depenada com cabeça, pés e vísceras comestíveis), de carcaça eviscerada (carcaça sem vísceras comestíveis), peito, perna (coxa + sobrecoxa), carne nobre (peito + coxa + sobrecoxa), fígado, coração, moela, gordura abdominal (gordura contida em volta da cloaca e dos músculos abdominais adjacentes) e da gordura de moela (gordura em volta da moela).

Para o cálculo de rendimento de carcaça e de carcaça eviscerada, tomou-se como base o peso vivo ao abate e, para determinação do rendimento dos outros cortes (peito, perna, carne nobre, fígado, moela, coração, gordura abdominal e gordura da moela), considerou-se o peso da carcaça depenada com pés, cabeça e vísceras comestíveis.

Tabela 2 - Composição das rações experimentais para os frangos de 4 a 21 dias de idade Table 2 - Ingredient and calculated compositions of the experimental diets from 4-21 days old

\begin{tabular}{|c|c|c|c|c|c|}
\hline \multirow[t]{2}{*}{$\begin{array}{l}\text { Ingrediente }(\%) \\
\text { Ingredient }\end{array}$} & \multicolumn{5}{|c|}{$\begin{array}{l}\text { Nível de inclusão do farelo de gérmen de milho desengordurado (FGMD), } \\
\text { Defatted corn germ meal (DCGM) inclusion level }\end{array}$} \\
\hline & 0 & 5 & 10 & 15 & 20 \\
\hline Milho (Corn) & 57,950 & 51,974 & 45,991 & 40,009 & 34,026 \\
\hline Farelo soja (Soybean meal) & 35,910 & 35,940 & 35,970 & 36,000 & 36,030 \\
\hline FGMD (DCGM) & - & 5,000 & 10,000 & 15,000 & 20,000 \\
\hline Óleo soja (Soybean oil) & 2,180 & 3,125 & 4,073 & 5,020 & 5,970 \\
\hline Fosfato bicálcio (Dicalcium phosphate) & 1,810 & 1,790 & 1,767 & 1,740 & 1,721 \\
\hline Calcário (Limestone) & 0,990 & 0,990 & 1,000 & 1,015 & 1,020 \\
\hline Sal comum (Salt) & 0,450 & 0,450 & 0,450 & 0,450 & 0,450 \\
\hline DL-metionina (99\%) (DL-methionine) & 0,060 & 0,086 & 0,108 & 0,130 & 0,152 \\
\hline L-lisina $\mathrm{HCl}(79 \%)$ (L-lysine $\mathrm{HCl})$ & 0,150 & 0,145 & 0,141 & 0,136 & 0,131 \\
\hline Suplemento vitamínico+mineral (Mineral + Vitamin supplement) & 0,500 & 0,500 & 0,500 & 0,500 & 0,500 \\
\hline Total & 100 & 100 & 100 & 100 & 100 \\
\hline \multirow{2}{*}{\multicolumn{6}{|c|}{$\begin{array}{l}\text { Valor calculado } \\
\text { Calculated value }\end{array}$}} \\
\hline & & & & & \\
\hline $\mathrm{EM}(M E)(\mathrm{kcal} / \mathrm{kg})$ & 2.959 & 2.962 & 2.965 & 2.968 & 2.971 \\
\hline $\mathrm{PB}(C P)(\%)$ & 21,500 & 21,500 & 21,500 & 21,500 & 21,500 \\
\hline $\mathrm{Ca}(\%)$ & 0,960 & 0,960 & 0,960 & 0,960 & 0,960 \\
\hline Fósforo disponível (\%) (Available phosphorus) & 0,450 & 0,450 & 0,450 & 0,450 & 0,450 \\
\hline Lisina total $(\%)$ (Total lysine) & 1,263 & 1,263 & 1,263 & 1,263 & 1,263 \\
\hline Metionina+Cistina total (\%) (Methionine+Total cystine) & 0,897 & 0,897 & 0,897 & 0,897 & 0,897 \\
\hline $\mathrm{FB}(C F)(\%)$ & 3,260 & 3,450 & 3,550 & 3,700 & 3,850 \\
\hline $\mathrm{EE}(\%)($ Fat $)$ & 4,657 & 5,418 & 6,180 & 6,940 & 7,700 \\
\hline
\end{tabular}

Suplemento vitamínico e mineral por kg de produto: Vit. A 2.400.000 UI; Vit. $D_{3} 440.000$ UI; Vit. E 6.000 mg; Vit. $K_{3} 500$ mg; Vit. $B_{1} 440$ mg; Vit. $B_{2} 1.200$ mg; Vit. $B_{6} 660 \mathrm{mg}$; Vit. $B_{12} 3.200 \mathrm{mg}$; Niacina 10.600 mg; Ac. Pantotênico 2.600 mg, Ac. Fólico 200 mg; Biotina 22 mg; Manganês 15.000 mg; Zinco 14.000 mg; Ferro 10.000 mg; Cobre 1.700 mg; lodo 300 mg; Selênio 50 mg; Cobalto 40 mg; Metionina 326.700 mg; Lisina 3.960 mg; Colina 84.000 mg; Veículo q.s.q - $1.000 \mathrm{~g}$.

Mineral and vitamin supplement. Nutritional levels per kg of product: Vit. A 2,400,000IU; Vit. $D_{3} 440,000 I U ;$ Vit. E 6,000 mg; Vit. $K_{3} 500 \mathrm{mg} ;$ Vit. $B_{1} 440 \mathrm{mg} ;$ Vit. $B_{2} 1.200 \mathrm{mg} ;$ Vit. $B_{6} 660 \mathrm{mg} ;$ Vit. $B_{12}$ 3,200 mg; Niacin 10,600 mg; Pantothenic acid 2,600 mg; Folic acid 200 mg; Biotin 22 mg; Manganese 15,000 mg; Zinc 14,000 mg; Iron 10,000 mg; Copper 1, 700 mg; lodine 300 mg; Selenium 50 mg; Cobalt $40 \mathrm{mg}$; Methionine 326,700 mg; Lysine 3,960 mg; Choline 84,000 mg; vehicle q. s. p. - 1,000 g. 
Tabela 3 - Composição das rações experimentais para os frangos de 22 a 35 dias de idade Table 3 - Ingredient and calculated compositions of the experimental diets from 22 - 35 days old

Ingrediente $(\%)$

Ingredient
Nível de inclusão do farelo de gérmen de milho desengordurado (FGMD), \%

Nível de inclusão do farelo de germen de milho desengordurado
\[ \text { Defatted corn germ meal (DCGM) inclusion level } \]
Milho (Corn)

Farelo soja (Soybean meal)

FGMD (DCGM)

Óleo soja (Soybean oil)

Fosfato bicálcio (Dicalcium phosphate)

Calcário (Limestone)

Sal comum (Salt)

DL-metionina (99\%) (DL-methionine)

L-lisina $\mathrm{HCl}(79 \%)$ (L-lysine $\mathrm{HCl})$

Suplemento vitamínico+mineral (Mineral + Vitamin supplement)

Cloreto de colina (Choline chloride)

Total

\begin{tabular}{ccccc}
\hline 0 & 5 & 10 & 15 & 20 \\
\hline 63,627 & 57,778 & 51,931 & 46,084 & 40,236 \\
29,974 & 29,889 & 29,803 & 29,718 & 29,632 \\
- & 5,000 & 10,000 & 15,000 & 20,000 \\
2,664 & 3,594 & 4,523 & 5,452 & 6,381 \\
1,628 & 1,605 & 1,583 & 1,560 & 1,538 \\
0,894 & 0,905 & 0,916 & 0,926 & 0,937 \\
0,396 & 0,401 & 0,405 & 0,410 & 0,414 \\
0,025 & 0,037 & 0,050 & 0,062 & 0,075 \\
0,172 & 0,171 & 0,169 & 0,168 & 0,167 \\
0,500 & 0,500 & 0,500 & 0,500 & 0,500 \\
0,120 & 0,120 & 0,120 & 0,120 & 0,120 \\
100 & 100 & 100 & 100 & 100 \\
\hline
\end{tabular}

Valor calculado

Calculated value

\begin{tabular}{|c|c|c|c|c|c|}
\hline EM (kcal/kg) (Metabolizable energy) & 3.058 & 3.061 & 3.065 & 3.068 & 3.071 \\
\hline PB (\%) (Crude protein) & 19,300 & 19,300 & 19,300 & 19,300 & 19,300 \\
\hline $\mathrm{Ca}(\%)$ (Calcium) & 0,874 & 0,874 & 0,874 & 0,874 & 0,874 \\
\hline $\mathrm{Pd}(\%)$ (Available phosphorus) & 0,406 & 0,406 & 0,406 & 0,406 & 0,406 \\
\hline Lisina total $(\%)$ (Total lysine) & 1,156 & 1,156 & 1,156 & 1,156 & 1,156 \\
\hline Metionina + Cistina total $(\%)$ (Methionine + Total cystine) & 0,825 & 0,825 & 0,825 & 0,825 & 0,825 \\
\hline FB (\%) (Crude fiber) & 3,189 & 3,334 & 3,479 & 3,624 & 3,769 \\
\hline $\mathrm{EE}(\%)($ Fat $)$ & 5,076 & 5,836 & 6,596 & 7,355 & 8,115 \\
\hline
\end{tabular}

Suplemento vitamínico e mineral por kg de produto (Mineral and vitamin supplement. Nutritional levels per kg of product): Vit. A 2.400 .000 UI; Vit. D 440.000 UI; Vit. E 6.000 mg; Vit. $K_{3} 500$ mg; Vit. B 440 mg; Vit. B 1.200 mg; Vit. B 660 mg; Vit. B 123.200 mg; Niacina (Niacin) 10.600 mg; Ac. pantotênico (Pantothenic acid) 2.600 mg, Ac. fólico (Folic acid) 200 mg; Biotina (Biotin) 22 mg; Mn 15.000 mg; Zn 14.000 mg; Fe 10.000 mg; Cu 1.700 mg; I 300 mg; Se 50 mg; Co 40 mg; Metionina (Methionine) 326.700 mg; Lisina (Lysine) 3.960 mg; Colina (Choline) 84.000 mg; Veículo q.s.q (vehicle q. s. p.) -1.000 g.

Tabela 4 - Composição das rações experimentais para os frangos na fase de 36 a 42 dias de idade Table 4 - Ingredient and calculated compositions of the experimental diets from 36-42 days old

Ingrediente $(\%) \quad$ Nível de inclusão do farelo de gérmen de milho desengordurado (FGMD), \%
Ingredient

Defatted corn germ meal (DCGM) inclusion level

\begin{tabular}{|c|c|c|c|c|c|}
\hline & & & & & \\
\hline & 0 & 5 & 10 & 15 & 20 \\
\hline Milho (Corn) & 66,506 & 60,656 & 54,811 & 48,961 & 43,113 \\
\hline Farelo soja (Soybean meal) & 26,621 & 26,536 & 26,450 & 26,365 & 26,280 \\
\hline FGMD (DCGM) & - & 5,000 & 10,000 & 15,000 & 20,000 \\
\hline Óleo soja (Soybean oil) & 3,556 & 4,486 & 5,415 & 6,345 & 7,274 \\
\hline Fosfato bicálcio (Dicalcium phosphate) & 1,426 & 1,404 & 1,381 & 1,359 & 1,335 \\
\hline Calcário (Limestone) & 0,991 & 1,002 & 1,012 & 1,023 & 1,034 \\
\hline Sal comum (Salt) & 0,213 & 0,218 & 0,222 & 0,227 & 0,232 \\
\hline DL-metionina (99\%) (DL-methionine) & 0,047 & 0,059 & 0,071 & 0,084 & 0,096 \\
\hline L-lisina $\mathrm{HCl}(79 \%)$ (L-lysine $\mathrm{HCl})$ & 0,140 & 0,139 & 0,138 & 0,136 & 0,135 \\
\hline Suplemento vitamínico+mineral (Mineral + Vitamin supplement) & 0,300 & 0,300 & 0,300 & 0,300 & 0,300 \\
\hline Cloreto de colina (Choline chloride) & 0,200 & 0,200 & 0,200 & 0,200 & 0,200 \\
\hline Total & 100 & 100 & 100 & 100 & 100 \\
\hline
\end{tabular}

Valor calculado

Calculated value

\begin{tabular}{|c|c|c|c|c|c|}
\hline $\mathrm{EM}(M E)(\mathrm{kcal} / \mathrm{kg})$ & 3.158 & 3.161 & 3.164 & 3.167 & 3.171 \\
\hline $\mathrm{PB}(C P)(\%)$ & 18,000 & 18,000 & 18,000 & 18,000 & 18,000 \\
\hline $\mathrm{Ca}(\%)$ & 0,850 & 0,850 & 0,850 & 0,850 & 0,850 \\
\hline Fósforo disponível (\%) (Available phosphorus) & 0,365 & 0,365 & 0,365 & 0,365 & 0,365 \\
\hline Lisina total $(\%)$ (Total lysine) & 1,040 & 1,040 & 1,040 & 1,040 & 1,040 \\
\hline Metionina + Cistina total $(\%)$ (Methionine + Total cystine) & 0,742 & 0,742 & 0,742 & 0,742 & 0,742 \\
\hline $\mathrm{FB}(C F)(\%)$ & 3,027 & 3,172 & 3,317 & 3,462 & 3,607 \\
\hline $\operatorname{EE}(\%)$ (Fat) & 6,032 & 6,792 & 7,552 & 8,312 & 9,073 \\
\hline
\end{tabular}

Suplemento vitamínico e mineral por kg de produto (Mineral and vitamin supplement. Nutritional levels per kg of product): Vit. A 833.300 UI; Vit. $\mathrm{D}_{3} 233.324$ UI; Vit. E 233.324 mg; Vit. $K_{3} 233.33$ mg; Vit. B 2.000 mg; Niacina (Niacin) 8.666 mg; Ac. pantotênico (Pantothenic acid) 3.000 mg, Mn 25.000 mg; Zn 16.667 mg; Fe 1.666 mg; Cu 2.666 mg; I 250 mg; Se 6.667 mg; Metionina (Methionine) 310 mg; Colina (Choline) 93.330 mg; Veículo q.s.q (vehicle q. s. p.) - 1.000 g. 
A viabilidade e o índice de eficiência econômica da inclusão do FGMD nas rações foram calculados considerando os custos das rações e de ração por quilograma de peso vivo, segundo Bellaver et al. (1985). Posteriormente, foram calculados os índices de eficiência econômica (IEE) e de custo (IC), segundo Barbosa et al. (1992). O custo médio da ração foi obtido considerando-se os preços dos ingredientes na região de Londrina, no mês junho de 2004.

$\mathrm{Na}$ análise estatística, foram utilizados os dados de desempenho e de carcaça e os índices econômicos, que foram analisados por meio do programa SAEG (UFV, 1998). Para avaliar os efeitos dos níveis de FGMD, foram feitas análises de variância e de regressão polinomial, considerando-se até o efeito quadrático. Para avaliar o efeito do sexo e das interações, foram realizadas análises de variância.

\section{Resultados e Discussão}

Os resultados da composição bromatológica (Tabela 5) diferiram quanto aos percentuais de PB, FB, EE e CIN em comparação aos encontrados por Butolo et al. (1998), Brum et al. (1999) e Rodrigues et al. (2001a).

$\mathrm{O}$ valor de $\mathrm{FB}$ foi, em média, 35\% inferior aos descritos por Soares et al. (2004) e 34\% superior aos reportados por Butolo et al. (1998) e Brum et al. (1999). O percentual de EE determinado neste experimento também foi inferior aos encontrados na literatura (Butolo et al., 1998; Brum et al., 1999; Rodrigues et al., 2001a). Segundo Bath et al. (1999), as variações na composição química do FGMD e do milho podem ser conseqüência das diferenças no clima, no solo, no cultivo, nas variedades e nos métodos de processamento.

Os coeficientes de metabolização de MS (CMMS), FB (CMFB), CP (CMPB) eEE (CMEE) do FGMD foram, respectivamente, de 60,$99 ; 16,73 ; 53,80$ e $82,71 \%$. O valor de $\mathrm{EB}$ foi de $3.602 \mathrm{kcal} / \mathrm{kg}$ de $\mathrm{MN}$, próximo ao verificado por Soares et al. (2004), de $3.584 \mathrm{kcal} / \mathrm{kg}$ de MN, e Brum et al. (1999), de $3.689 \mathrm{kcal} / \mathrm{kg}$ de MN. O valor de $\operatorname{EMA}_{\mathrm{n}}(2.413$ $\mathrm{kcal} / \mathrm{kg}$ de $\mathrm{MN}$ ) foi semelhante ao descrito por Brum et al. (1999), de $2.468 \mathrm{kcal}$ de EMAn/kg de MN.

Os valores de EMAn do FGMD obtidos com 20 e $40 \%$ de substituição da ração-referência foram $2.454 \mathrm{e}$ $2.372 \mathrm{kcal} / \mathrm{kg}$ de $\mathrm{MN}$, respectivamente, e se assemelham aos relatados por Butolo et al. (1998), que obtiveram 2.364 e $2.256 \mathrm{kcal} / \mathrm{kg}$ de $\mathrm{MN}$ nos níveis de 20 e $40 \%$ de substituição, respectivamente.

A média dos valores de EMAn (2.413 kcal EMAc/ $\mathrm{kg}$ de MN) foi 28,4\% inferior à descrita por Rostagno et al. (2000), de $3.371 \mathrm{kcal} / \mathrm{kg}$ de matéria natural para o grão do milho.
Os dados de desempenho (ganho peso, consumo de ração e conversão alimentar) dos frangos de corte, em cada período avaliado, são apresentados na Tabela 6 .

Não foram observados efeitos significativos $(\mathrm{P}>0,05)$ das interações nível de inclusão do FGMD $\times$ sexo dos frangos sobre as características avaliadas nas diferentes fases. No período de 4 a 21 dias de idade, o ganho de peso (GP) e o consumo de ração (CR) apresentaram resposta linear crescente e a conversão alimentar (CA), reposta linear decrescente, ao aumento do nível de FGMD nas rações experimentais. As equações foram: $\hat{Y}=0,641+0,003 X$ $\left(\mathrm{R}^{2}=0,92\right)$ para GP; $\hat{\mathrm{Y}}=0,929+0,0018 \mathrm{X}\left(\mathrm{R}^{2}=0,71\right)$ para $\mathrm{CR}$; e $\hat{Y}=1,450-0,0037 X\left(R^{2}=0,67\right)$ para CA. Portanto, nesta fase, quanto maior a inclusão do FGMD nas rações, maiores os valores de GP e CR e melhores os de CA.

$\mathrm{O}$ aumento no ganho de peso dos frangos alimentados com os níveis crescentes de FGMD provavelmente foi ocasionado pelo acréscimo no consumo das rações, o que pode estar relacionado aos maiores níveis de óleo, pois a substituição de carboidrato por gordura em rações para frangos de corte têm grandes vantagens sobre os parâmetros de desempenho (Junqueira et al., 2005; Dell'Isola et al., 2003).

A conversão alimentar dos frangos também melhorou com a inclusão do FGMD nas rações, provavelmente em

Tabela 5 - Composição química, coeficiente de metabolização da MS (CMMS), da PB (CMPB), da FB (CMFB) e do EE (CMEE) e valores energéticos do farelo de gérmen de milho desengordurado (FGMD), na matéria natural

Table 5 - Chemical composition, coefficient of metabolization of DM (DMMC), CP (CPMC), CF (CFMC), and EE (EEMC) and values of defatted corn germ meal energy (DCGM), as-fed basis

\begin{tabular}{lc}
\hline Item (\%) & $\begin{array}{c}\text { Valores } \\
\text { Values }\end{array}$ \\
Item (\%) & 89,44 \\
MS (DM) & 0,60 \\
EE & 9,81 \\
PB (CP) & 5,29 \\
FB (CF) & 6,44 \\
CIN (Mineral matter) & 60,99 \\
CMMS (DMMC) & 53,80 \\
CMPB (CPMC) & 16,73 \\
CMFB (CFMC) & 82,71 \\
CMEE (EEMC) & 3.602 \\
EB (GE) (kcal/kg) & \\
\hline EMA (kcal/kg), na matéria natural & \\
Corrected apparent metabolizable energy (kcal/kg), & \\
as-fed basis & 2.454 \\
\hline FGMD (20\%) (DCGM) & 2.372 \\
FGMD (40\%) (DCGM) & 2.413 \\
\hline Média & \\
Mean &
\end{tabular}


razão da adição de óleo, que, possivelmente, favoreceu a digestibilidade (Junqueira et al., 2005; Dell'Isola et al., 2003).

$\mathrm{Na}$ fase de 4 a 42 dias de idade, observou-se efeito linear $(\mathrm{P}<0,01)$ em relação às características avaliadas. As equações de regressão foram: $\mathrm{GP}=2,193+0,0073 \mathrm{X}$ $\left(\mathrm{R}^{2}=0,95\right)$; $\mathrm{CR}=3,864+0,0074 \mathrm{X}\left(\mathrm{R}^{2}=0,91\right)$ e $\mathrm{CA}=1,764$ $0,002 X\left(R^{2}=0,92\right)$.

Portanto, à semelhança do período de 4 a 21 dias de idade, na análise de todo o período experimental, constatou-se melhora nas médias de GP, CR e CA para as inclusões de FGMD nas rações.

No período de 4 a 21 dias de idade, as fêmeas apresentaram médias de ganho de peso e consumo de ração superiores às dos machos. Porém, na fase de 4 a 42 dias, os machos foram superiores às fêmeas.

As médias observadas para as características de carcaça, em relação aos níveis de inclusão do FGMD, são apresentadas na Tabela 7. Aos 43 dias de idade, houve efeito significativo $(\mathrm{P}<0,05)$ para a inclusão do FGMD sobre os pesos de abate, da carcaça e da carcaça eviscerada. Na fase de 4 a 42 dias de idade, a inclusão do FGMD influenciou positivamente o ganho de peso e, conseqüentemente, determinou maiores pesos de abate, da carcaça e da carcaça eviscerada.

As equações de regressão determinadas foram: peso de abate $=2,340+0,004 \mathrm{X}\left(\mathrm{R}^{2}=0,52\right)$; peso da carcaça $=$ $2,047+0,003 X\left(R^{2}=0,59\right)$ e peso da carcaça eviscerada $=$ $1,909+0,003 X\left(\mathrm{R}^{2}=0,50\right)$.

Os rendimentos de carcaça, de carcaça eviscerada, de peito, de pernas e de carnes nobres e o peso de peito e de pernas, em relação ao peso de abate, não foram influenciados pelos tratamentos.

As médias das características de peso e de rendimento das vísceras e da gordura abdominal e da moela são descritas na Tabela 8.

As equações de regressão para peso e rendimento de coração foram: $\hat{Y}=0,0108+0,00007 X\left(R^{2}=0,55\right)$ e $\hat{\mathrm{Y}}=0,524+0,0028 \mathrm{X}\left(\mathrm{R}^{2}=0,51\right)$, respectivamente.

O peso de gordura da moela e da gordura abdominal, com seus respectivos rendimentos, não sofreram efeito significativo dos níveis crescentes de inclusão do FGMD na ração.

Os machos apresentaram médias superiores às das fêmeas para quase todas as características avaliadas.

Na Tabela 9 constam as médias de peso e rendimentos de carcaça, peito e perna, de acordo com o sexo dos frangos de corte. Para o peso e o rendimento de cortes, de vísceras e de gordura da carcaça, não foram observadas interações tratamentos $\times$ sexo.
As médias para peso de abate, de carcaça, de carcaça eviscerada, de peito e de pernas foram maiores para os machos que para as fêmeas. Estes resultados foram semelhantes aos observados por Figueiredo et al. (1998), Lisboa et al. (1999), Moreira et al. (2003) e Stringhini et al. (2003).

Os rendimentos de carcaça, carcaça eviscerada e carne nobre não diferiram $(\mathrm{P}>0,05)$ entre sexos, confirmando os relatos de Stringhini et al. (2003) e Moreira et al. (2003). Verificou-se, no entanto, efeito do sexo sobre os rendi-

Tabela 6 - Efeito dos tratamentos e do sexo sobre o ganho de peso (GP), o consumo de ração $(C R)$ e a conversão alimentar (CA) dos frangos de corte de 4 a 21 e de 4 a 42 dias de idade

Table 6 - Effect of treatments and sex on weight gain (WG), feed intake (FI) and feed:gain ratio (F/C) of broilers from 4-21 and 4-42 days old

\begin{tabular}{|c|c|c|c|}
\hline \multirow{2}{*}{$\begin{array}{l}\text { Tratamento } \\
\text { Treatment }\end{array}$} & \multicolumn{3}{|c|}{$\begin{array}{l}\text { Fator }(\mathrm{kg}) \\
\quad \text { Factor }\end{array}$} \\
\hline & $\mathrm{GP} \quad(W G)$ & $\mathrm{CR}(F I)$ & $\mathrm{CA}(F C)$ \\
\hline & \multicolumn{3}{|c|}{$\begin{array}{l}4 \text { a } 21 \text { dias de idade } \\
4-21 \text { days old }\end{array}$} \\
\hline $0 \quad(\%)$ & 0,647 & 0,920 & 1,42 \\
\hline $5 \quad(\%)$ & 0,647 & 0,946 & 1,46 \\
\hline $10(\%)$ & 0,678 & 0,957 & 1,41 \\
\hline $15(\%)$ & 0,680 & 0,947 & 1,39 \\
\hline $20(\%)$ & 0,707 & 0,964 & 1,36 \\
\hline $\begin{array}{l}\text { Efeito de regressão } \\
\text { Regression effect }\end{array}$ & $\begin{array}{l}\text { Linear** } \\
\text { Linear }\end{array}$ & $\begin{array}{l}\text { Linear** } \\
\text { Linear }\end{array}$ & $\begin{array}{l}\text { Linear** } \\
\text { Linear }\end{array}$ \\
\hline \multicolumn{4}{|l|}{$\begin{array}{l}\text { Sexo } \\
\text { Sex }\end{array}$} \\
\hline Fêmeas & $0,679^{\mathrm{a}}$ & $0,963^{\mathrm{a}}$ & 1,42 \\
\hline $\begin{array}{l}\text { Females } \\
\text { Machos } \\
\text { Males }\end{array}$ & $0,664^{\mathrm{b}}$ & $0,931^{b}$ & 1,40 \\
\hline \multirow[t]{2}{*}{ CV (\%) } & 3,361 & 2,644 & 3,711 \\
\hline & \multicolumn{3}{|c|}{$\begin{array}{c}4 \text { a } 42 \text { dias de idade } \\
4-42 \text { days old }\end{array}$} \\
\hline $0 \quad(\%)$ & 2,206 & 3,871 & 1,76 \\
\hline $5 \quad(\%)$ & 2,209 & 3,876 & 1,76 \\
\hline $10(\%)$ & 2,266 & 3,953 & 1,75 \\
\hline $15(\%)$ & 2,311 & 3,992 & 1,73 \\
\hline $20(\%)$ & 2,337 & 3,998 & 1,71 \\
\hline $\begin{array}{l}\text { Efeito de regressão } \\
\text { Regression effect }\end{array}$ & $\begin{array}{l}\text { Linear** } \\
\text { Linear }\end{array}$ & $\begin{array}{l}\text { Linear** } \\
\text { Linear }\end{array}$ & $\begin{array}{l}\text { Linear** } \\
\text { Linear }\end{array}$ \\
\hline $\begin{array}{l}\text { Sexo } \\
\text { Sex }\end{array}$ & & & \\
\hline $\begin{array}{l}\text { Fêmeas } \\
\text { Females }\end{array}$ & $2,103^{b}$ & $3,743^{b}$ & $1,78^{\mathrm{a}}$ \\
\hline $\begin{array}{l}\text { Machos } \\
\text { Males }\end{array}$ & $2,429^{\mathrm{a}}$ & $4,132^{\mathrm{a}}$ & $1,70^{\mathrm{b}}$ \\
\hline CV (\%) & 2,790 & 2,978 & 1,548 \\
\hline
\end{tabular}


Tabela 7 - Peso ao abate e pesos e rendimentos de carcaça, de carcaça eviscerada, de peito, perna e carne nobre de frangos de corte aos 43 dias de idade em função dos níveis de inclusão do farelo de gérmen de milho desengordurado (FGMD) na ração

Table 7 - Effects of treatments on weight at slaughter, weights and yields of carcass, eviscerated carcass, breast, leg, and prime meat of broilers at 43 days old according to increasing dietary defatted corn germ meal (DCGM) levels

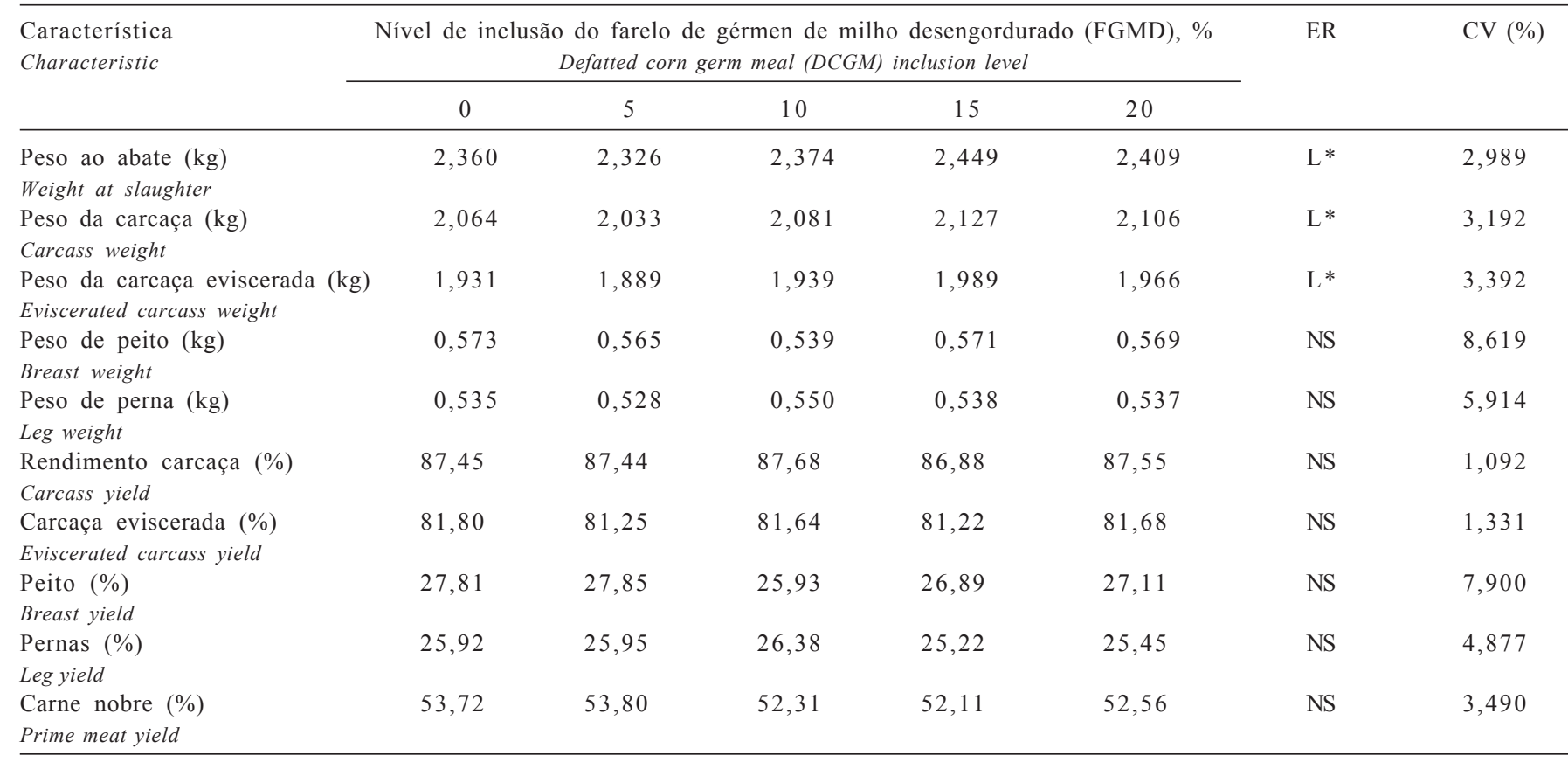

$\mathrm{CV}=$ coeficiente de variação.

$\mathrm{ER}=$ efeito regressão.

NS = não-significativo.

$L^{*}=$ efeito linear $(P<0,05)$.

$C V=$ coefficient of variation

$R E=$ regresion effect.

$N S=$ not significant .

$L^{*}=$ linear effect $(P<0.05)$

mentos de peito e perna. Confirmando os resultados descritos na literatura (Figueiredo et al., 1998; Moreira et al., 2003; Stringhini et al., 2003), as fêmeas apresentaram valores superiores de rendimento de peito e inferiores de rendimento de pernas em relação aos machos.

As médias dos pesos e rendimentos de vísceras comestíveis e gordura de machos e fêmeas são descritas na Tabela 10 .

Os machos apresentaram maiores médias de peso de fígado, moela e coração e rendimento de coração. Não houve diferenças significativas entre sexos para as médias de peso e rendimento da gordura da moela e rendimentos de fígado e moela. As fêmeas só apresentaram médias superiores para a característica gordura abdominal, corroborando os dados obtidos por Lisboa et al. (1999) e Moura et al. (2002). Segundo Moreira et al. (2003), o maior acúmulo de gordura abdominal pode estar relacionado à existência de adipócitos de maior tamanho nas fêmeas.

Constam na Tabela 11 os resultados da análise econômica, com simulação de preço do FGMD de $45,7 \%$ do valor do milho, índice normalmente utilizado no mercado.

No período de 4 a 21 dias de idade, não houve efeito $(\mathrm{P}>0,05)$ dos tratamentos experimentais sobre o ICM e IEE.
Porém, quando se analisou o período experimental de 4 a 42 dias de idade, foi observado aumento linear crescente para o ICM ( $\left.\hat{Y}=100,084+0,108 X, R^{2}=0,90\right)$ e linear decrescente para IEE ( $\left.\hat{Y}=99,984-0,106 X, R^{2}=0,98\right)$, para os níveis crescentes de inclusão FGMD (X) na ração.

A ração-referência promoveu os melhores índices de eficiência econômica e de custo e, portanto, a inclusão do FGMD na ração não é economicamente viável.

Considerando os resultados de desempenho, observou-se possibilidade da inclusão de até $20 \%$ de FGMD na ração dos frangos.

\section{Conclusões}

$\mathrm{O}$ farelo de gérmen de milho desengordurado apresentou coeficientes de metabolização de MS, FB, PB e EE de 60,99; 16,$73 ; 53,80$ e $82,71 \%$, respectivamente, e valor médio de EMAn de $2.413 \mathrm{kcal} / \mathrm{kg}$ na matéria natural. A inclusão de FGMD na ração de frangos de corte na proporção de até $20 \%$ da ração não comprometeu o desempenho produtivo e as características de carcaça, porém, pelos preços que este alimento foi obtido, não foi economicamente viável. 
Tabela 8 - Efeitos dos níveis de farelo de gérmen de milho desengordurado (FGMD) na ração sobre as características de peso (g) e rendimento (\%) de fígado, moela, coração, gordura da moela e gordura abdominal dos frangos em relação à carcaça

Table 8 - Effects of increasing dietary defatted corn germ meal (DCGM) levels on weight (g) and yields (\%) of liver, gizzard, heart, gizzard fat, and abdominal fat in relation to broiler carcass

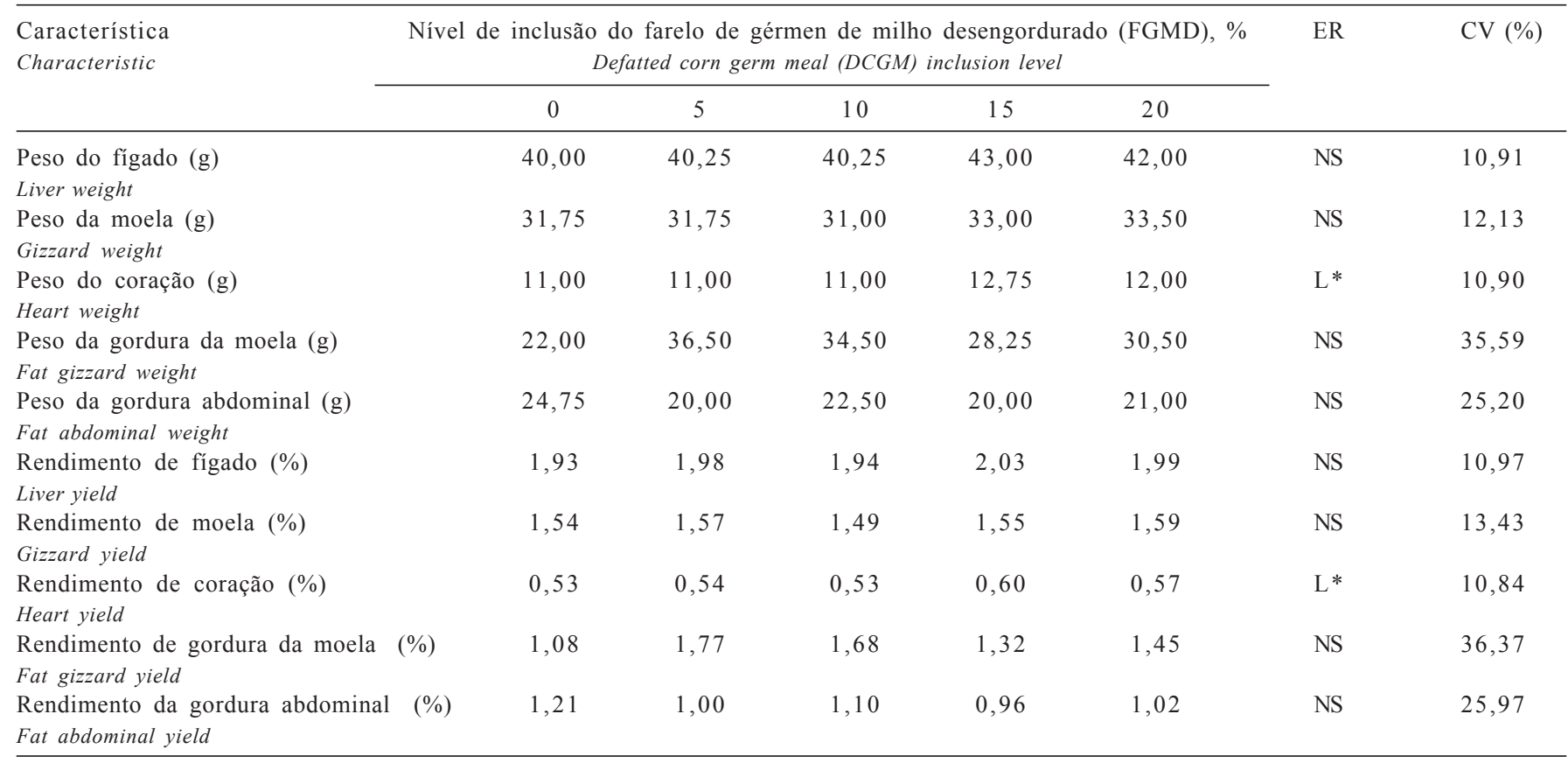

$\mathrm{CV}=$ coeficiente de variação (CV = coefficient of variation).

$\mathrm{ER}=$ efeito regressão $(R E=$ regresion effect $)$.

$\mathrm{NS}=$ não-significativo (NS $=$ not significant $)$.

$L^{*}=$ efeito linear $(P<0,05)\left(L^{*}=\right.$ linear effect $\left.[P<0.05]\right)$

Tabela 9 - Médias dos pesos (g) e dos rendimentos (\%) das características de carcaça de frangos de corte de acordo com o sexo

Table 9 - Means of weight $(g)$ and yield (\%) carcass characteristics of broilers according to sex

\begin{tabular}{|c|c|c|c|}
\hline \multirow[t]{2}{*}{$\begin{array}{l}\text { Característica } \\
\text { Characteristic }\end{array}$} & \multicolumn{2}{|c|}{$\begin{array}{c}\text { Sexo } \\
\text { Sex }\end{array}$} & \multirow[t]{2}{*}{ CV (\%) } \\
\hline & $\begin{array}{l}\text { Fêmea } \\
\text { Female }\end{array}$ & $\begin{array}{c}\text { Macho } \\
\text { Male }\end{array}$ & \\
\hline $\begin{array}{l}\text { Peso de abate } \\
\text { Weight at slaughter }\end{array}$ & $2.196^{\mathrm{b}}$ & $2.570^{\mathrm{a}}$ & 2,99 \\
\hline $\begin{array}{l}\text { Peso da carcaça } \\
\text { Carcass weight }\end{array}$ & $1.926^{\mathrm{b}}$ & $2.239^{\mathrm{a}}$ & 3,19 \\
\hline $\begin{array}{l}\text { Peso da carcaça eviscerada } \\
\text { Eviscerated carcass weight }\end{array}$ & $1.790^{\mathrm{b}}$ & $2.096^{\mathrm{a}}$ & 3,39 \\
\hline $\begin{array}{l}\text { Peso de peito } \\
\text { Breast weight }\end{array}$ & $0,537^{b}$ & $0,590^{\mathrm{a}}$ & 8,61 \\
\hline $\begin{array}{l}\text { Peso de perna } \\
\text { Leg weight }\end{array}$ & $0,488^{\mathrm{b}}$ & $0,586^{\mathrm{a}}$ & 5,91 \\
\hline $\begin{array}{l}\text { Rendimento de carcaça } \\
\text { Carcass yield }\end{array}$ & 87,60 & 87,12 & 1,09 \\
\hline $\begin{array}{l}\text { Rendimento de carcaça eviscerada } \\
\text { Eviscerated carcass yield }\end{array}$ & 81,50 & 81,54 & 1,33 \\
\hline $\begin{array}{l}\text { Rendimento de peito } \\
\text { Breast yield }\end{array}$ & $27,89^{\mathrm{a}}$ & $26,34^{b}$ & 7,90 \\
\hline $\begin{array}{l}\text { Rendimento de pernas } \\
\text { Leg yield }\end{array}$ & $25,35^{\mathrm{b}}$ & $26,21^{\mathrm{a}}$ & 4,88 \\
\hline $\begin{array}{l}\text { Rendimento de cortes nobres } \\
\text { Prime meat yield }\end{array}$ & 53,25 & 52,56 & 3,49 \\
\hline
\end{tabular}

$\mathrm{CV}=$ coeficiente de variação

$a, b=$ médias seguidas de letras diferentes namesmalinha são diferentes $(P<0,05)$.

$\mathrm{CV}=$ coefficient of variation.

$a, b=$ means within a row followed by different letters differ $(P<0.05)$
Tabela 10 - Médias dos pesos (g) e dos rendimentos (\%) das vísceras e gordura dos frangos de acordo com o sexo

Table 10 - Means of weights $(\mathrm{g})$ and yields (\%) of viscera and fat of broilers according to sex

\begin{tabular}{|c|c|c|c|}
\hline \multirow{2}{*}{$\begin{array}{l}\text { Característica } \\
\text { Characteristic }\end{array}$} & \multicolumn{2}{|c|}{$\begin{array}{l}\text { Sexo } \\
\text { Sex }\end{array}$} & \multirow[t]{2}{*}{ CV (\%) } \\
\hline & $\begin{array}{l}\text { Fêmea } \\
\text { Female }\end{array}$ & $\begin{array}{c}\text { Macho } \\
\text { Male }\end{array}$ & \\
\hline $\begin{array}{l}\text { Peso do fígado } \\
\text { Liver weight }\end{array}$ & $37,40^{\mathrm{b}}$ & $43,80^{\mathrm{a}}$ & 10,91 \\
\hline $\begin{array}{l}\text { Peso da moela } \\
\text { Gizzard weight }\end{array}$ & $30,40^{\mathrm{b}}$ & $34,00^{\mathrm{a}}$ & 12,13 \\
\hline $\begin{array}{l}\text { Peso do coração } \\
\text { Heart weight }\end{array}$ & $10,20^{\mathrm{b}}$ & $12,90^{\mathrm{a}}$ & 10,90 \\
\hline $\begin{array}{l}\text { Peso da gordura da moela } \\
\text { Fat gizzard weight }\end{array}$ & 28,80 & 31,90 & 35,59 \\
\hline $\begin{array}{l}\text { Peso da gordura abdominal } \\
\text { Fat abdominal weight }\end{array}$ & $24,70^{\mathrm{a}}$ & $18,60^{\mathrm{b}}$ & 25,20 \\
\hline $\begin{array}{l}\text { Rendimento de fígado } \\
\text { Liver yield }\end{array}$ & 1,99 & 1,96 & 10,97 \\
\hline $\begin{array}{l}\text { Rendimento de moela } \\
\text { Gizzard yield }\end{array}$ & 1,58 & 1,52 & 13,43 \\
\hline $\begin{array}{l}\text { Rendimento de coração } \\
\text { Heart yield }\end{array}$ & $0,53^{b}$ & $0,57^{\mathrm{a}}$ & 10,84 \\
\hline $\begin{array}{l}\text { Rendimento da gordura da moela } \\
\text { Fat gizzard yield }\end{array}$ & 1,49 & 1,43 & 36,37 \\
\hline $\begin{array}{l}\text { Rendimento da gordura abdominal } \\
\text { Fat abdominal yield }\end{array}$ & $1,28^{\mathrm{a}}$ & $0,83^{b}$ & 25,97 \\
\hline
\end{tabular}

CV = coeficiente de variação

$a, b=$ médias seguidas de letras diferentes namesma linha são diferentes $(P<0,05)$. $\mathrm{CV}=$ coefficient of variation

$a, b=$ means within a row followed by different letters differ $(P<0.05)$. 
Tabela 11 - Custo médio em ração por quilograma de peso vivo ganho (R\$/kg de PV), índice médio de custo (IMC) e índice de eficiência econômica (IEE) dos frangos de corte de acordo com os níveis de farelo de gérmen de milho desengordurado (FGMD) ${ }^{1}$ na ração

Table 11 - Mean diet cost per kilogram of body weight gain (R\$/kg of BW), mean cost index (MCl) and economic efficiency index (EEl) of broilers according to the increasing dietary defatted corn germ meal levels (DCGM)

Item

Item
Nível de inclusão do farelo de gérmen de milho desengordurado (FGMD), \% Defatted corn germ meal (DCGM) inclusion level

$\begin{array}{llll}0 & 5 & 10 & 15\end{array}$

20

4 a 21 dias de idade

$$
\text { 4-21 days old }
$$

Custo da ração $(\mathrm{R} \$ \mathrm{~kg})$

Diet cost $(R \$ / \mathrm{kg})$

Custo em ração ( $\mathrm{R} \$ / \mathrm{kg}$ de $\mathrm{PV})$

Cost in diet $(R \$ / \mathrm{kg}$ of $B W)$

ICM $(M C I)$

IEE (EEI)

\section{0,736}

1,04
0,746

1,09
0,755

1,06
104,02

95,89

101,87

101,87
97,93

100,00

4 a 42 dias de idade

$$
\text { 4-42 days old }
$$

15

(

Custo da ração $(\mathrm{R} \$ / \mathrm{kg})$

Diet cost ( $R \$ / \mathrm{kg})$

Custo em ração ( $\mathrm{R} \$ \mathrm{~kg}$ de $\mathrm{PV})$

Cost in diet $(R \$ / \mathrm{kg}$ of $B W)$

ICM $(M C I)^{2}$

IEE $(E E I)^{2}$

\section{0,735}

1,29

100,00

100,00
0,743

1,309

100,54

99,47
0,751

1,31

101,08

98,94
0,764

0,772

1,06

1,05

101,52

98,26

100,50

99,38

1 Considerando o preço do FGMD é igual a $45,77 \%$ do preço do milho grão (Considering DCGM price of $45.7 \%$ of corn grain price).

2 Efeito linear (Linear effect).

\section{Literatura Citada}

ALBINO, L.F.T.; FERREIRA, A.S.; FIALHO, E.T. et al. Determinação dos valores de energia metabolizável e matéria seca aparentemente metabolizável de alguns alimentos. Revista da Sociedade Brasileira de Zootecnia, v.11, n.2, p.207-220, 1982.

ANDRIGUETTO, J.M; PERLY, L.; MINARDI, I. et al. Nutrição animal: As bases e os fundamentos da nutrição e os alimentos. 2.ed. São Paulo: Nobel, 1983. 425p.

BARBOSA, H.P.; FIALHO, E.T.; FERREIRA, A.S. et al. Triguilho para suínos nas fases inicial de crescimento, crescimento e terminação. Revista Brasileira de Zootecnia, v.21, n.5, p.827-837, 1992.

BATH, D.B.; DUNBRAR, J.; KING, J. et al. Byproducts and unusual feedstuffs. Feedstuffs, v.71, n.4, 1999.

BELLAVER, C.; FIALHO, E.T.; FROTAS, J.F.S et al. Radícula de malte na alimentação de suínos em crescimento e terminação. Pesquisa Agropecuária Brasileira, v.20, n.8, p.969-974, 1985 .

BRUM, P.A.R.; ZANOTTO, D.L.; LIMA, G.J.M.M. et al. Determinação dos valores da composição química e da energia metabolizável de ingredientes para aves. Revista Brasileira de Ciência Avícola, v.1, n.3, p.187-192, 1999.

BUTOLO, E.A.F.; NOBRE, P.T.C.; BOTELHO, F.G.A. et al. Determinação do valor energético e nutritivo de gérmen de milho desengordurado para frangos de corte. In: CONFERÊnCIA APINCO'98, 1998, Campinas. Anais... Campinas: Fundação APINCO de Ciência e Tecnologia Avícolas, 1998. p.40.

DELL'ISOLA, A.T.P.; VELOSO, J.A.F.; BAIÃO, N.C. et al. Efeito do soja em dietas com diferentes níveis de cálcio sobre a absorção e retenção óssea de cálcio e de fósforo em frangos de corte. Arquivo Brasileiro de Medicina Veterinária, v. $55, \mathrm{n} .4$, p.461-466, 2003.
DERMACHI, J.J.A.A. Bovinos leiteiros. In: SIMPÓSIO DE NUTRIÇÃO ANIMAL, 1., 1998, Espírito Santo do Pinhal. Anais... Espírito Santo do Pinhal: Fundação Pinhalense de Ensino, 1998. p.117-130.

FIGUEIREDO, A.C.S.; SOARES, P.R.; ALBINO, L.F.T. et al. Desempenho, rendimento de carcaça e avaliação econômica de diferentes programas de restrição alimentar em frangos de corte. Revista Brasileira de Zootecnia, v.27, n.3, p.564-571, 1998.

INSTITUTO ADOLFO LUTZ. Normas analíticas do Instituto Adolfo Lutz. 3.ed. São Paulo: Instituto Adolfo Lutz, 1985. v.1. $375 \mathrm{p}$.

JUNQUEIRA, O.M; ANDREOTTI, M.; ARAUJO, L.F. et al. Valor energético de algumas fontes lipídicas determinado com frangos de corte. Revista Brasileira de Zootecnia, v.34, n.6, p.23352339, 2005 (supl).

LISBOA, J.S.; SILVA, J.; SILVA, M.A. et al. Rendimento de carcaça de três grupos genéticos de frangos de corte alimentados com rações contendo diferentes teores de proteína. Revista Brasileira de Zootecnia, v.28, n.3, p.548-554, 1999.

MATTERSON, L.D.; POTTER, L.M.; STUTUZ, N.W.; SINGSEN, E.P. The metabolizable energy of feed ingredients for chickens. Research Report, n.7, p.3-11, 1965.

MOREIRA, I.; RIBEIRO, C.R.; FURLAN, A.C. et al. Utilização do farelo de germe de milho desengordurado na alimentação de suínos em crescimento e terminação. Revista Brasileira de Zootecnia, v.31, n.6, p.2238-2246, 2002.

MOREIRA, J.; MENDES, A.A.; GARCIA, E.A. Avaliação de desempenho, rendimento de carcaça e qualidade da carne do peito em frangos de linhagens de conformação versus convencionais. Revista Brasileira de Zootecnia, v.32 n.6, p.1663-1673, 2003.

RODRIGUES, P.B.; ROSTAGNO, H.S.; ALBINO, L.F.T. et al. Valores energéticos do milheto, do milho e subprodutos do milho, determinados com frangos de corte e galos adultos. Revista Brasileira de Zootecnia, v.30, n.6, p.1767-1777, 2001a (supl). 
RODRIGUES, P.B.; ROSTAGNO, H.S.; ALBINO, L.F.T. et al. Aminoácidos digestíveis verdadeiros do milheto, do milho e subprodutos do milho, determinados com galos adultos cecectomizados. Revista Brasileira de Zootecnia, v.30, n.6, p.2046-2058, 2001b (supl.).

ROSTAGNO, H.S.; ALBINO, L.F.T.; DONZELE, J.L. et al. Tabelas brasileiras para aves e suínos (Composição de alimentos e exigências nutricionais). Viçosa, MG: Universidade Federal de Viçosa, 2000. p.141.

ROSTAGNO, H.S. 2003. Farelo de gérmen de milho nas rações de frangos de corte. Disponível em: $<$ http://www.Polinutri.com.br Acesso em: 11/04/2003.

SOARES, L.P.S.; SILVA, C.A.; PINHEIRO, J.W. et al. Farelo de gérmen de milho desengordurado na alimentação de suínos em crescimento e terminação. Revista Brasileira Zootecnia, v.33, n.6, p.1768-1776, 2004.

STRINGHINI, J.H.; LABOISSIERE, M.; MURAMATSU, K. Avaliação do desempenho e rendimento de carcaça e quatro linhagens de frangos de corte criados em Goiás. Revista Brasileira de Zootecnia, v.32, n.1, p.183-190, 2003.

UNIVERSIDADE FEDERAL DE VIÇOSA - UFV. SAEG - Sistema de análises estatísticas e genéticas: Versão 7.1. Viçosa, MG: 1998. (Manual do Usuário). 